\title{
Mesenchymal stem cells from skin lesions of psoriasis patients promote proliferation and inhibit apoptosis of HaCaT cells
}

\author{
R.F. Liu, F. Wang, Q. Wang, X.C. Zhao and K.M. Zhang \\ Institute of Dermatology, Taiyuan City Centre Hospital, Taiyuan, Shanxi Province, \\ China
}

Corresponding author: K.M. Zhang

E-mail: luyaoliu@126.com

Genet. Mol. Res. 14 (4): 17758-17767 (2015)

Received August 12, 2015

Accepted October 16, 2015

Published December 22, 2015

DOI http://dx.doi.org/10.4238/2015.December.21.49

\begin{abstract}
Psoriasis is an inflammatory skin disease characterized by excessive proliferation and abnormal differentiation and apoptosis of keratinocytes (KCs). Mesenchymal stem cells (MSCs) from skin lesions of psoriasis patients demonstrate abnormal cytokine secretion, which may affect KC proliferation and apoptosis. Here, we explored how MSCs from skin lesions of psoriasis patients affect $\mathrm{HaCaT}$ cell proliferation and apoptosis. First, flow cytometry and multipotent differentiation methods were used to identify skin MSCs, which were then co-cultured with $\mathrm{HaCaT}$ cells. HaCaT cell proliferation was analyzed in real-time, and cell cycle progression and apoptosis were assessed by flow cytometry. Cell morphologies and multipotencies of skin MSCs were similar between the psoriasis group and healthy control group, with high levels of CD29, CD44, CD73, CD90, and CD105 and limited expression of CD34, CD45, and HLA$\mathrm{DR}$. MSCs from skin lesions of psoriasis patients promote $\mathrm{KC}$ proliferation more potently and are less capable of inducing KC apoptosis. This may
\end{abstract}


underlie KC proliferation and abnormal apoptosis in psoriasis skin lesions, which results in abnormal thickening of the epidermis.

Key words: Apoptosis; HaCaT cell; Mesenchymal stem cell; Keratinocyte proliferation; Psoriasis; Skin lesions

\section{INTRODUCTION}

Psoriasis is a chronic inflammatory and proliferative skin disease (Nickoloff and Nestle, 2004). Histologically, epidermal acanthosis is one of the characteristic pathological changes, which is associated with both excessive proliferation and abnormal apoptosis of epidermal keratinocytes (KCs) (Rückert et al., 2000; Boehm, 2006; Chan et al., 2006; Raj et al., 2006; Tse et al., 2008). The proliferation and differentiation of epidermal KCs is regulated by a complex network of growth factors and cytokines, including epidermal growth factor, insulin-like growth factor-I, transforming growth factor $\alpha$, interleukin (IL)-1, IL-6, and tumor necrosis factor (TNF)- $\alpha$ (Rückert et al., 2000). Normal expression levels of these cytokines are essential for maintaining epidermal homeostasis. Apoptosis of epidermal KCs is another important aspect of maintaining epidermal homeostasis. The body adjusts KC proliferation and regulates the growth and differentiation of epidermal cells through apoptosis.

In preliminary studies, several abnormal biological features have been found in bone marrow and skin lesion mesenchymal stem cells (MSCs) of psoriasis patients (Zhang et al., 2010; Hou et al., 2013, 2014; Liu et al., 2013, 2014). Skin MSCs (S-MSCs) are an important component of the microenvironment of the skin. These cells are not only involved in skin tissue development and regeneration, but can also significantly affect the skin microenvironment through the secretion of cytokines (Salvolini et al., 2010). Indeed, complex cytokine networks in skin lesions of psoriasis patients play important roles in psoriasis pathogenesis (Jain et al., 2009; Blumberg et al., 2010; Guilloteau et al., 2010). A previous study found abnormal cytokine secretion in S-MSCs around psoriatic lesions (Orciani et al., 2011). As psoriasis patients exhibit abnormal epidermal KC proliferation and apoptosis (Rückert et al., 2000; Chan et al., 2006), it is possible that skin lesion MSCs may influence KC proliferation and apoptosis.

Therefore, in our study, skin lesion MSCs from psoriasis patients and human KC (HaCaT) cells were co-cultured to investigate how the former affect the cell cycle progression and apoptosis of the latter. Our results confirmed the hypothesis that skin lesion MSCs from psoriasis patients enhance proliferation and reduce apoptosis in HaCaT cells. This may be one of the reasons for the abnormal proliferation and reduced apoptosis of the epidermal KCs of the patients, which eventually leads to epidermal acanthosis.

\section{MATERIAL AND METHODS}

\section{Subjects}

All patients and volunteers gave informed consent for their participation in the study. The protocols involving human subjects were approved by the Medical Ethics Committee of Taiyuan City Centre Hospital and were in accordance with the 1964 Helsinki declaration and its later amendments or comparable ethical standards.

Eight outpatients (4 female patients and 4 male patients; age range: 21-54 years; mean 
age \pm SD: $38.13 \pm 1.22$ years) from the Institute of Dermatology, Taiyuan City Centre Hospital, and 8 sex- and age-matched volunteers from the Urology and Plastic Surgery Department, were enrolled in this study. The volunteers showed no signs of systemic disease in a routine health examination. The patients were diagnosed with psoriasis vulgaris based on both the appearance of the skin and skin lesion shave biopsies. They had a 20-day to 20 -year disease course, with 15 to $70 \%$ plaque coverage. Psoriasis was scored based on the Psoriasis Area and Severity Index (PASI), which measures the average redness, thickness, and scaliness of the lesions (each graded on a 0-4 scale), weighted by the area of involvement (head and neck, trunk, upper, and lower extremities). All patients had scores between 5.1 and 25.9, with an average score of $13.54 \pm 7.08$. From these 8 patients, 4 patients had progressive stage psoriasis and 4 were in the stationary stage. No subjects had received corticosteroid hormones, tretinoin, immunosuppressants, or phototherapy within 6 months before enrollment.

\section{Reagents}

Cell culture plates and Transwell chambers were purchased from Corning Incorporated (Corning, NY, USA). DMEM/F12 medium, DMEM/High Glucose medium, B-27 supplement, and fetal bovine serum (FBS) were purchased from Invitrogen (Grand Island, NY, USA). Trypsin and dispase enzyme II were purchased from Sigma-Aldrich (St. Louis, MO, USA). The DNA PreP Reagents kit and Annexin V-FITC cell apoptosis analysis kit were purchased from BeckmanCoulter (Los Angeles, CA, USA). A Real Time Cell Analysis instrument was purchased from ACEA Biosciences (San Diego, CA, USA). An IMT2 inverted phase-contrast microscope was obtained from Olympus (Tokyo, Japan), and an EPICS-XL Flow Cytometer FACSCalibur was purchased from BeckmanCoulter. The HaCaT cell line was obtained from Cell Line Services (Heidelberg, Germany).

\section{S-MSC separation, cultivation, and identification}

S-MSCs were prepared as previously described (Liu et al., 2013, 2014). Briefly, psoriatic skin lesion and normal skin specimens (8 cases each) were minced and digested with $0.25 \%$ dispase enzyme II. The dermis was collected and the cells were separated. Cells were then cultured in T25 flasks at a density of $1 \times 10^{5} / \mathrm{cm}^{2}$ at $37^{\circ} \mathrm{C}$. At nearly $90 \%$ confluence, the cells were digested with $0.25 \%$ trypsin and passaged. At passage 5, $2 \times 10^{5}$ cells from psoriatic skin lesions and healthy individuals were transferred to tubes and incubated with PE- or FITC-labeled antibodies against the following human S-MSC surface markers: CD29, CD44, CD73, CD90, CD105, CD34, CD45, and HLA-DR. Cells were then analyzed by two-color flow cytometry (EPICS-XL Flow Cytometer FACSCalibur).

S-MSCs from both groups were induced at passage 5 to differentiate into lipocytes, osteoblasts, or chondrocytes. After adipogenic differentiation for 10 days, cells were fixed with $10 \%$ formalin, washed with $60 \%$ isopropyl alcohol, and stained with oil red O. After osteogenic differentiation for 3 weeks, cells were fixed with $10 \%$ formalin and stained with $2 \%$ alizarin red solution. After chondrogenic differentiation for 21 days, micromasses were fixed with $4 \%$ paraformaldehyde, embedded in optimal cutting temperature compound, cut into 5- $\mu \mathrm{m}$ sections, and stained with toluidine blue. Negative controls, in which differentiation-inducing supplements were omitted, were included for each differentiation assay. 


\section{HaCaT cell cultivation}

HaCaT cells were cultured in DMEM/High Glucose medium with 10\% FBS and incubated at $37^{\circ} \mathrm{C}$, with $5 \% \mathrm{CO}_{2}$ and saturated humidity. When cells had grown to nearly $90 \%$ confluency, they were digested with $0.25 \%$ trypsin and passaged. Cells in logarithmic growth phase were used for research.

\section{HaCaT cell proliferation curve in S-MSC co-culture}

We co-cultivated S-MSCs with HaCaT cells and performed real-time cell analysis using a Real Time Cell Analysis instrument (ACEA Biosciences) to evaluate HaCaT cell proliferation. In our preliminary experiment, culture medium (DMEM/High Glucose medium with $10 \%$ FBS) was added to an E-Plate and background resistance was measured. HaCaT cells in the logarithmic growth phase were inoculated on an E-Plate L8 $\left(3 \times 10^{4}\right.$ cells/well, cultivated in DMEM/High Glucose medium with $10 \%$ FBS) with passage-5 S-MSCs from one patient and from one control $\left(3 \times 10^{4}\right.$ cells/well, cultivated in DMEM/F12 medium with 10\% FBS) in the upper chamber (well diameter: $0.4 \mu \mathrm{m}$ ). Monocultured wells were used to determine "basal" HaCaT cell proliferation (DMEM/ F12 medium with 10\% FBS only, in the upper chamber). The plate was kept on a clean bench at room temperature for $30 \mathrm{~min}$, and then placed on the station and cultivated at $37^{\circ} \mathrm{C}$ with $5 \%$ $\mathrm{CO}_{2}$ and saturated humidity. A real-time dynamic cell proliferation test was performed for $96 \mathrm{~h}$, according to the manufacturer instructions and the maximum cell index (CIMax) was determined. Data are reported as the average of measurements performed in duplicate. The appropriate time for detection of cell cycle progression and apoptosis was determined by assessing the time of maximum and minimum cell proliferation from proliferation curves.

\section{Cell cycle determination of HaCaT cells in S-MSC co-culture by flow cytometry}

HaCaT cells in logarithmic growth phase were diluted to $1 \times 10^{5}$ cells $/ \mathrm{mL}$ in DMEM/High Glucose medium with $10 \%$ FBS, and $2 \times 10^{5}$ cells were added to each lower chamber of a 12-well Transwell plate, with a pore diameter of $0.4 \mu \mathrm{m}$. Passage- 5 psoriatic or normal S-MSCs $\left(2 \times 10^{5}\right.$ cells, cultivated in S-MSC complete medium) were added to the upper chambers of the Transwell plate $(\mathrm{N}=8$ replicates). Eight chambers were used for monoculture of HaCaT cells (commensurable S-MSC complete medium was added to the upper chambers). Each measurement was performed in duplicate, and data are reported as the average. The plate was incubated at $37^{\circ} \mathrm{C}$ in a humidified incubator supplemented with $5 \% \mathrm{CO}_{2}$. After incubation for $48 \mathrm{~h}$ (as per our preliminary Real Time Cell Analysis experiment), the upper chamber with the S-MSCs was removed. HaCaT cells in the lower chambers were digested with $0.25 \%$ trypsin and collected. HaCaT cells were washed twice with cold PBS (with centrifugation at $200 \mathrm{~g}$ for $5 \mathrm{~min}$ in between washes), and were diluted to $1 \times 10^{6} \mathrm{cells} / \mathrm{mL}$. Then, $100 \mu \mathrm{L}$ of sample was added to $200 \mu \mathrm{L}$ DNA PreP Reagent, and mixed immediately. After static incubation in the dark at room temperature for $10 \mathrm{~s}, 1 \mathrm{~mL}$ DNA PreP Stain was added to the cells and mixed gently. Samples were incubated for $30 \mathrm{~min}$ in the dark. Cells were filtered through a 300-mesh filter and the cell cycle phase was detected by flow cytometry.

\section{Determination of the apoptotic ratio of HaCaT cells in S-MSC co-culture}

To determine the apoptotic ratio in HaCaT cells, cells were co-cultured with S-MSCs as discussed above. After incubation for $72 \mathrm{~h}$ (as per our preliminary Real Time Cell Analysis 
experiment), the upper chamber with the S-MSCs was removed. HaCaT cells in the lower chambers were then digested with $0.25 \%$ trypsin and collected. HaCaT cells were washed twice with cold PBS (with centrifugation at $400 \mathrm{~g}$ for $5 \mathrm{~min}$ in between washes). Approximately $5 \times 10^{5}$ cells were resuspended in $100 \mu \mathrm{L}$ binding buffer and thoroughly mixed with $5 \mu \mathrm{L}$ Annexin V-FITC followed by addition of $2.5 \mu \mathrm{L}$ propidium iodide (PI). After mixing, cells were incubated in the dark on ice for $10 \mathrm{~min}$. Cells were mixed with $400 \mu \mathrm{L}$ binding buffer, and the apoptotic ratio was analyzed by flow cytometry. The percentage of cells that were Annexin $\mathrm{V}^{+}$was defined as the apoptotic ratio. The percentage of cells that were Annexin $\mathrm{V}^{+} / \mathrm{PI}^{-}$was considered as the early apoptotic ratio and the percentage of cells that were Annexin $\mathrm{V}^{+} / \mathrm{PI}^{+}$was defined as the mid/late apoptotic ratio.

\section{Statistical analysis}

Data are reported as means \pm SD. For HaCaT cell proliferation and apoptotic ratio tests, the differences between groups were examined by single-factor analysis of variation and differences between means were assessed using the Student-Newman-Keuls test. Analysis was performed with the SPSS16.0 software (SPSS Inc., Chicago, IL, US). The correlation of S-phase cell proportion and the apoptotic ratio of $\mathrm{HaCaT}$ cells in the patients group with severe psoriasis was analyzed by Pearson correlation. A P value $<0.05$ was considered to be statistically significant.

\section{RESULTS}

\section{Identification and morphology of S-MSCs from psoriasis patients and controls}

As observed through an inverted microscope, the morphology of S-MSCs from the psoriasis group and from the control group was similar. Flow cytometric analysis of surface antigens of S-MSCs at passage 5 from both groups showed high expression levels of CD29, CD44, CD73, CD90, and CD105, and low expression of CD34, CD45, and HLA-DR. The S-MSCs all differentiated into the relevant cells and tissues after adipogenic, osteogenic, and chondrogenic inductions, indicating that the isolated and cultured cells meet the identification criteria for MSCs (Dominici et al., 2006).

\section{Real-time cell analysis}

Compared with HaCaT monocultures, HaCaT cells co-cultured with S-MSCs showed an increase in proliferation. Both S-MSCs from psoriasis patients and from healthy controls promoted HaCaT cell proliferation (CIMax value of monoculture: 4.70, CIMax value of co-culture with patient's skin lesion MSCs: 6.67, CIMax value of co-culture with control MSCs: 5.70$)$, but skin lesion MSCs showed a stronger effect on proliferation than control S-MSCs (Figure 1). After 32-60 $\mathrm{h}$ of cultivation, cells of the 3 groups successively reached their maximum proliferation states. Cell counts gradually decreased after this time (all showed different degrees of apoptosis or cell death) and the cell count of the monoculture group dropped to its minimum value at about $76 \mathrm{~h}$.

\section{HaCaT cell cycle test}

Upon Annexin-V/PI staining, HaCaT cells co-cultured with S-MSCs exhibited a decline in the proportion of G0-G1 phase cells in comparison to the monocultured group, whereas the 
proportion of cells in $S$ phase increased $(P<0.05)$. Compared with the control group, the proportion of cells that entered the $S$ phase was increased in the patient group $(P<0.05)$, as shown in Figure 2 and Table 1. These results suggest that S-MSCs can promote HaCaT cell proliferation, and that MSCs originating from skin lesions show the greatest induction of proliferation.

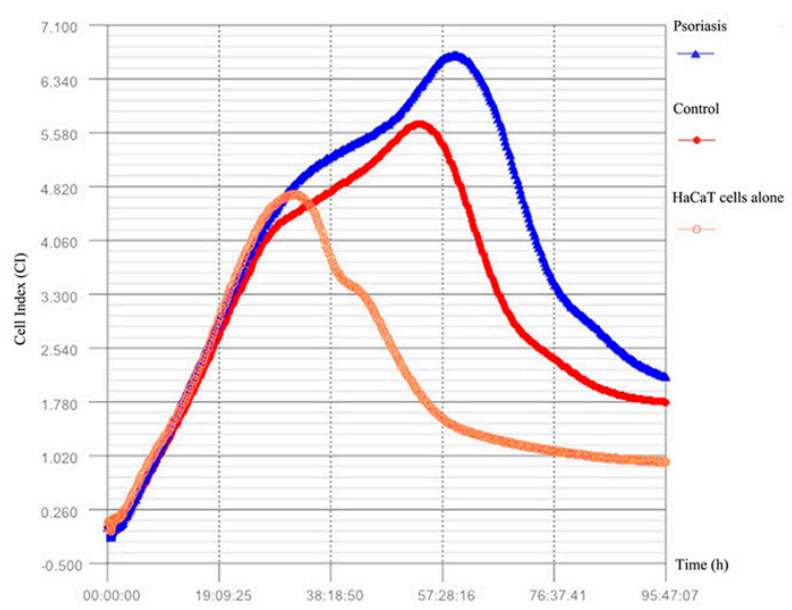

Figure 1. HaCaT cell growth curves. Growth curves of monocultured HaCaT cells (orange), HaCaT cells co-cultured with control S-MSCs (red), and HaCaT cells co-cultured with psoriatic S-MSCs (blue).
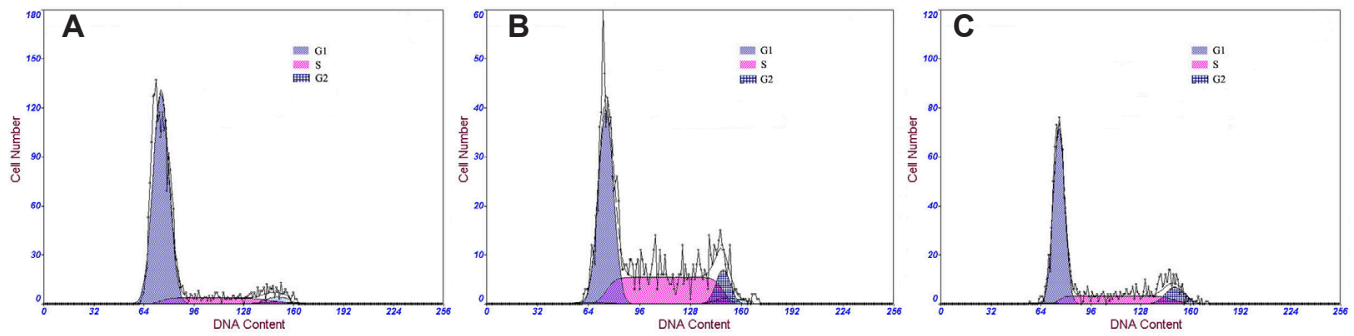

Figure 2. HaCaT cell cycle. A. The percentage of S phase cells for monocultured $\mathrm{HaCaT}$ cells is $13.7 \%$; B. that for HaCaT cells co-cultured with psoriatic S-MSCs is $41.9 \%$; and C. that for HaCaT cells co-cultured with control S-MSCs is $24 \%$.

Table 1. Comparison of the cell cycle of skin lesion MSCs from psoriasis patients and HaCaT co-cultures, control S-MSCs HaCaT co-cultures, and HaCaT monocultures (means $\pm \mathrm{SD}$ ).

\begin{tabular}{lcccc}
\hline Group & No. of cases & \multicolumn{3}{c}{ Phase distribution of cell cycle (\%) } \\
\cline { 3 - 5 } & & G0/G1 & S & G2/M \\
\hline HaCaT monoculture & 8 & $86.87 \pm 3.59$ & $7.35 \pm 3.14$ & $5.77 \pm 4.25$ \\
Co-culture with patient MSCs & 8 & $47.38 \pm 4.22^{\star \S}$ & $42.78 \pm 2.61^{\star \S}$ & $9.86 \pm 4.35^{\dagger \neq}$ \\
Co-culture with control MSCs & 8 & $55.45 \pm 1.37^{*}$ & $33.88 \pm 7.40^{\star}$ & $10.68 \pm 7.24^{\dagger}$ \\
\hline
\end{tabular}

*Statistically significant compared to monoculture group (all $\mathrm{P}$ values $<0.05$ ). \$Statistically significant compared to control group $(P<0.05)$. ${ }^{\dagger}$ Not statistically significant compared to monoculture group (all $P$ values $\left.>0.05\right)$. ${ }^{\ddagger}$ Not statistically significant compared to control group $(P>0.05)$. 


\section{HaCaT cell apoptotic ratios}

After co-culturing HaCaT cells with S-MSCs from psoriasis patients and controls, we found that MSCs from both psoriasis skin lesions and from healthy skin can induce apoptosis of HaCaT cells $(P<0.05)$. However, the skin lesion MSCs showed a reduced ability to induce apoptosis $(P<$ 0.05), as shown in Figure 3 and Table 2.
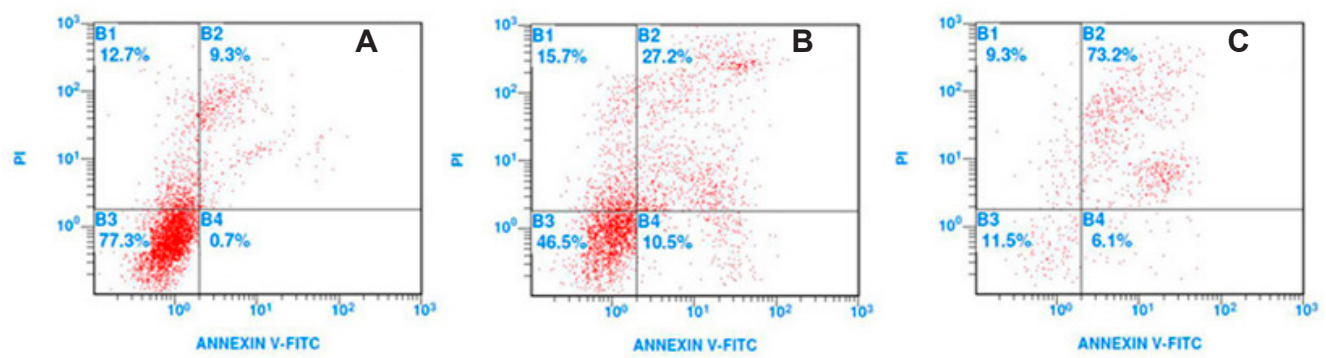

Figure 3. Apoptosis of HaCaT cells. A. The apoptosis rate of monocultured $\mathrm{HaCaT}$ cells is $10 \%$; $\mathbf{B}$. that of $\mathrm{HaCaT}$ cells co-cultured with psoriatic S-MSCs is $37.7 \%$; and C. that of HaCaT cells co-cultured with control S-MSCs is $79.3 \%$.

Table 2. Comparison of the apoptotic ratio of skin lesion MSCs from psoriasis patients and HaCaT co-cultures, control S-MSCs and HaCaT co-cultures, and HaCaT monocultures (means \pm SD).

\begin{tabular}{lcc}
\hline Group & Number of cases & Apoptotic ratio of HaCaT cells (\%) \\
\hline HaCaT monoculture & 8 & $22.00 \pm 10.30$ \\
Co-culture with patient MSCs & 8 & $37.19 \pm 15.35^{\star}$ \\
Co-culture with control MSCs & 8 & $75.44 \pm 8.41^{*}$ \\
\hline
\end{tabular}

*Statistically significant compared to monoculture group (all $P$ values $<0.05$ ). \$Statistically significant compared to control group $(P<0.05)$.

\section{Correlation between S phase cell proportion and HaCaT cell apoptotic ratio in the patients group and the psoriasis PASI score}

Pearson correlation analysis showed that the proportion of HaCaT cells in the $\mathrm{S}$ phase in the psoriasis patients group did not correlate with the PASI score $(r=-0.132, \mathrm{P}=0.833)$. The apoptotic ratio of these cells was also not correlated with the PASI score $(r=0.302, \mathrm{P}=0.468)$.

\section{DISCUSSION}

In this study, we explored how MSCs from skin lesions of psoriasis patients affect HaCaT cell proliferation and apoptosis, in an attempt to understand the role of MSCs in the pathogenesis of psoriasis. We found that S-MSCs from lesions of patients promote KC proliferation more potently and exhibit attenuated induction of KC apoptosis, compared to S-MSCs from control individuals.

Cell proliferation is a basic biological process important for reproduction, ontogenesis, and repair. Cell apoptosis, also referred as programmed cell-death, is equally important during the development of multicellular organisms (Abrams et al., 1993). Proliferation and apoptosis are genetically and ontogenetically complementary processes. KCs represent a major human 
epidermal cell type. Since they are highly proliferative and differentiative, they play an important role in skin rejuvenation and immunity. Cells in the basal layer constantly divide, proliferate, and are lifted upward as they age. They pass through the prickle cell layer, reach the outermost granular layer, and eventually become horny layer cells. During the differentiation process from the epidermal basal cell layer to the horny layer, KCs finally lose their nuclei and corresponding organelles (keratinization). Many researchers consider terminal differentiation as a special type of apoptosis.

The organism adjusts $\mathrm{KC}$ proliferation in the skin through apoptosis and regulation of epidermal growth and differentiation, so as to generate the horny layerand maintain epidermal homeostasis. The processes of KC proliferation, differentiation, and apoptosis (formation of the horny layer) drives the cells to gradually differentiate, mature, and die. These processes are precisely controlled by multiple inter-constrained regulating mechanisms, as any abnormalities that affect KC proliferation and apoptosis will affect homeostasis and result in complex pathophysiological response, for example the onset and development of diseases such as psoriasis.

$\mathrm{HaCaT}$ cells originate from adult epidermal cells. In this study, compared with the monoculture group, the proportion of $\mathrm{HaCaT}$ cells that entered $\mathrm{S}$ phase after co-cultivation with healthy control S-MSCs was increased. The proportion of HaCaT cells in S phase was $7.35 \pm 3.14 \%$ in the monoculture group and $33.88 \pm 7.40 \%$ in the control S-MSCs co-culture group $(P<0.05)$. In a cell cycle, DNA synthesis and histone-DNA assembly into chromatin are completed in $S$ phase. Thus, a higher proportion of cells in $S$ phase indicates that more cells have entered the material preparation process to prepare for cell division. It is therefore an important sign of increased cellular proliferative ability. Our results indicate that MSCs can promote HaCaT cell proliferation. Moreover, S-MSCs can also induce HaCaT apoptosis. The apoptotic ratio of the monoculture group was $22.00 \pm 10.30 \%$, and the apoptotic ratio of the cells co-cultured with healthy control S-MSCs was $75.44 \pm 8.41 \%$. The dynamic balance between promoting proliferation and inducing apoptosis is essential for maintaining the natural growth balance of KCs and epidermal homeostasis.

Psoriasis is a common chronic inflammatory skin disease that severely affects the quality of life through spontaneous mitigation and aggravation. It is a lifelong disease with a multifactorial cause, including genetic factors and immunization (Lowes et al., 2007). Epidermal acanthosis and parakeratosis caused by hyperproliferation and abnormal differentiation of KCs are typical histological features of psoriasis (Schön and Boehncke, 2005; Tse et al., 2008; Xu et al., 2013). Proliferation and abnormal differentiation of psoriatic KCs may be caused by many reasons. For example, miR-203 (Sonkoly et al., 2007), miR-125b (Xu et al., 2011), miR-424 (Ichihara et al., 2011), and miR-99a (Lerman et al., 2011) are all involved in abnormal proliferation and differentiation of psoriatic KCs. Downregulation of CCHCR1 expression in basal-layer KCs may cause abnormal proliferation of psoriatic epidermal cells (Tiala et al., 2008), while IL-22 (and its related cytokines IL19, IL-20, and IL-24) causes epidermal hyperplasia (Boniface et al., 2005; Sa et al., 2007). Elevated expression levels of P2X7RB in psoriatic skin lesions result in increased proliferation and reduced apoptosis of skin lesion KCs (Killeen et al., 2013). Moreover, the epidermis of skin lesions inhibits KC apoptosis through elevated expression of IL-15 (Rückert et al., 2000). KCs in psoriatic skin lesions show resistance to apoptosis, and lack of human pro-apoptotic gene (P53) activity (Qin et al., 2002). Increased proliferation and reduced apoptosis of psoriatic epidermal KCs (Laporte et al., 2000) lead to further development of proliferative and hypertrophic skin lesions. However, to date there have been no studies on the effects of psoriatic skin lesion MSCs on KC proliferation and apoptosis.

This study shows that, compared with the control group, the proportion of S phase HaCaT cells was increased upon co-cultivation with MSCs from psoriasis skin lesions, indicating that MSCs from psoriasis skin lesions enhance KC proliferation. In addition, our results also showed 
that reduced KC apoptosis in psoriasis patients is closely related to skin lesion MSCs. These effects of S-MSCs on KC apoptosis may be one of the reasons for abnormal KC apoptosis in psoriasis. The increased proliferative ability and reduced apoptotic ability conferred by psoriasis skin lesion MSCs on KCs eventually results in abnormal proliferation of epidermal cells. The $\mathrm{S}$ phase cell proportion and apoptotic rate of $\mathrm{HaCaT}$ cells from the patient group did not correlate with the psoriasis PASI score, suggesting that increased proliferation and inhibited apoptosis of skin lesion KCs are caused by abnormalities of the MSCs per se, and are not significantly associated with systemic inflammatory responses. The exact mechanism by which skin lesion MSCs cause increased proliferation and inhibited apoptosis of KCs is an important topic for future studies.

An in-depth study of the regulatory pathways and factors involved in KC proliferation and apoptosis is of considerable theoretical and practical significance, and can facilitate new insights into the treatment of this disease. Drugs that treat psoriasis by targeting KC apoptosis are currently available. One example is infliximab, a chimeric anti-TNF- $\alpha$ antibody that induces apoptosis of skin lesion KCs so as to achieve rapid and sustained treatment of plaque psoriasis (Krüger-Krasagakis et al., 2006). In addition, the induction of KC apoptosis by using narrow spectrum ultraviolet radiation $\mathrm{b}$ is a therapeutic option for psoriasis vulgaris.

This study provides new insights on psoriasis pathogenesis and will serve as a reference in searching for new drug targets and methods for treating this disease.

\title{
Conflicts of interest
}

The authors declare no conflict of interest.

\section{ACKNOWLEDGMENTS}

\author{
Research supported by the National Natural Science Foundation of China (\#NFSC \\ 81271768 and \#81472888).
}

\section{REFERENCES}

Abrams JM, White K, Fessler LI and Steller H (1993). Programmed cell death during Drosophila embryogenesis. Development 117: 29-43.

Blumberg H, Dinh H, Dean CJr, Trueblood ES, et al. (2010). IL-1RL2 and its ligands contribute to the cytokine network in psoriasis. J. Immunol. 185: 4354-4362.

Boehm I (2006). Apoptosis in physiological and pathological skin: implications for therapy.Curr. Mol. Med. 6: $375-394$.

Boniface K, Bernard FX, Garcia M, Gurney AL, et al. (2005). IL-22 inhibits epidermal differentiation and induces proinflammatory gene expression and migration of human keratinocytes. J. Immunol. 174: 3695-3702.

Chan JR, Blumenschein W, Murphy E, Diveu C, et al. (2006). IL-23 stimulates epidermal hyperplasia via TNF and IL-20R2dependent mechanisms with implications for psoriasis pathogenesis. J. Exp. Med. 203: 2577-2587.

Dominici M, Le Blanc K, Muellerl, Slaper-Cortenbach I, et al. (2006). Minimal criteria for defining multipotent mesenchymal stromal cells. The International Society for Cellular Therapy position statement. Cytotherapy 8: 315-317.

Guilloteau K, Paris I, Pedretti N, Boniface K, et al. (2010). Skin inflammation induced by the synergistic action of IL-17A, IL-22,

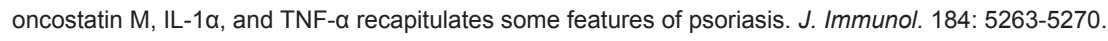

Hou R, Yin G, An P, Wang C, et al. (2013). DNA methylation of dermal MSCs in psoriasis: identification of epigenetically dysregulated genes. J. Dermatol. Sci. 72: 103-109.

Hou R, Liu R, Niu X, Chang W, et al. (2014). Biological characteristics and geneexpression pattern of bone marrow mesenchymal stem cells in patients with psoriasis. Exp. Dermatol. 23: 521-523.

Ichihara A, Jinnin M, Yamane K, Fujisawa A, et al. (2011). microRNA-mediated keratinocyte hyperproliferation in psoriasis vulgaris. Br J. Dermatol. 165: 1003-1010. 
Jain S, Kaur IR, Das S, Bhattacharya SN, et al. (2009). T helper 1 to T helper 2 shift in cytokine expression: an autoregulatory process in superantigen-associated psoriasis progression? J. Med. Microbiol. 58: 180-184.

Killeen ME, Ferris L, Kupetsky EA, Falo L Jr, et al. (2013). Signaling through purinergic receptorsfor ATP induces human cutaneous innate and adaptive Th17 responses: implications in the pathogenesis of psoriasis. J. Immunol. 190: 4324-4336.

Krüger-Krasagakis S, Galanopoulos VK, Giannikaki L, Stefanidou M, et al. (2006). Programmed cell death of keratinocytes in infliximab-treated plaque-type psoriasis. Br J. Dermatol. 154: 460-466.

Laporte M, Galand P, Fokan D, de Graef C, et al. (2000). Apoptosis in established andhealing psoriasis. Dermatology 200: 314-316.

Lerman G, Avivi C, Mardoukh C, Barzilai A, et al. (2011). MiRNA expression inpsoriatic skin: reciprocal regulation of hsa-miR99a and IGF-1R. PLoS One 6:e20916.

Liu R, Yang Y, Yan X and Zhang K (2013). Abnormalities in cytokine secretion frommesenchymal stem cells in psoriatic skin lesions. Eur. J. Dermatol. 23: 600-607.

Liu R, Wang Y, Zhao X, Yang Y, et al. (2014). Lymphocyte inhibition is compromised inmesenchymal stem cells from psoriatic skin. Eur. J. Dermatol. 24: 560-567.

Lowes MA, Bowcock AM and Krueger JG (2007). Pathogenesis and therapy of psoriasis. Nature 445: 866-873.

Nickoloff BJ and Nestle FO (2004). Recent insights into the immunopathogenesis of psoriasis provide new therapeutic opportunities. J. Clin. Invest. 113: 1664-1675.

Orciani M, Campanati A, Salvolini E, Lucarini G, et al. (2011). The mesenchymal stemcell profile in psoriasis. Br J. Dermatol. 165: 585-592.

Qin JZ, Chaturvedi V, Denning MF, Bacon P, et al. (2002). Regulation of apoptosis by p53 in UV-irradiated human epidermis, psoriatic plaques and senescent keratinocytes. Oncogene 21: 2991-3002.

Raj D, Brash DE and Grossman D (2006). Keratinocyte apoptosis in epidermal development and disease. J. Invest. Dermatol. 126: 243-257.

Rückert R, Asadullah K, Seifert M, Budagian VM, et al. (2000). Inhibition of keratinocyte apoptosis by IL-15: a new parameter in the pathogenesis of psoriasis? J. Immunol. 165: 2240-2250.

Sa SM, Valdez PA, Wu J, Jung K, et al. (2007). The effects of IL-20 subfamily cytokines on reconstituted human epidermis suggest potential roles in cutaneous innate defense and pathogenic adaptive immunity in psoriasis. J. Immunol. 178: 2229-2240.

Salvolini E, Orciani M, Vignini A, Mattioli-Belmonte M, et al. (2010). Skin-derived mesenchymalstem cells (S-MSCs) induce endothelial cell activation by paracrine mechanisms. Exp. Dermatol. 19: 848-850.

Schön MP and Boehncke WH (2005). Psoriasis. N. Engl. J. Med. 352: 1899-1912.

Sonkoly E, Wei T, Janson PC, Sääf A, et al. (2007). MicroRNAs: novel regulators involved in the pathogenesis of psoriasis? PLoS One 2: e610.

Tiala I, Wakkinen J, Suomela S, Puolakkainen P, et al. (2008). The PSORS1 locus gene CCHCR1 affects keratinocyte proliferation in transgenic mice. Hum. Mol. Genet. 17: 1043-1051.

Tse WP, Cheng CH, Che CT and Lin ZX (2008). Arsenic trioxide, arsenic pentoxide, and arsenic iodide inhibit human keratinocyte proliferation through the induction of apoptosis. J. Pharmacol. Exp. Ther. 326: 388-394.

Xu N, Brodin P, Wei T, Meisgen F, et al. (2011). MiR-125b, a microRNA downregulated in psoriasis, modulates keratinocyte proliferation by targeting FGFR2. J. Invest. Dermatol. 131: 1521-1529.

Xu N, Meisgen F, Butler LM, Han G, et al. (2013). MicroRNA-31 is overexpressed in psoriasis and modulates inflammatory cytokine and chemokine production in keratinocytes via targeting serine/threonine kinase 40. J. Immunol. 190: 678-688.

Zhang K, Liu R, Yin G, Li X, et al. (2010). Differential cytokine secretion of cultured bone marrow stromal cells from patients with psoriasis and healthy volunteers. Eur. J. Dermatol. 20: 49-53. 